\title{
Pollution des laits de femme par les résidus de pesticides organochlorés en France ${ }^{*}$
}

\author{
par \\ F. M. LUQUET, J. GOURSAUD et J. CASALIS \\ Laboratoire d'Industrie Laitière, 13, rue de l'Université \\ 59509 Douai
}

Dans différents pays, la pollution du lait de femme par les résidus de pesticides a fait l'objet de nombreuses études, mais en France les données étaient fragmentaires et imprécises.

Le Laboratoire d'Industrie Laitière de Douai a effectué pendant une année (novembre 1971 à décembre 1972) une enquête à partir d'échantillons de lait qui lui ont été fournis par dix lactariums situés dans différentes villes de France, à savoir : Bordeaux, Lille 1 et 2, Lyon, Marmande, Nantes, Paris, Rouen, Saint-Etienne et Strasbourg. Ces lactariums collectent au total 65 à 700001 de lait de femme par an (moyenne 10,4 1 par mère nourrice).

Les résultats de l'enquête sont résumés dans le tableau I et dans la figure 1. L'examen de ces documents permet les constatations suivantes :

$1^{\circ}$ La pollution des échantillons est en général très importante et dépasse de très loin les tolérances préconisées dans les réglementations de l'Organisation Mondiale de la Santé.

$2^{\circ}$ Les laits de femme en France sont surtout pollués par le DDT et ses métabolites (pp'DDE : 2 à 4 ppm et pp'DDT : 2 ppm), par le $\mathrm{HCH}$ sous ses différentes formes (isomères $\alpha, \beta$ et $\gamma$ : au total taux supérieur à $1 \mathrm{ppm}$ ), pár le $\mathrm{HCB}$, et a un moindre degré par l'Heptachlore époxyde et la Dieldrine.

$3^{\circ}$ La comparaison des moyennes nationales des taux de pollution des laits de vache (déterminés par des enquêtes précédemment

* Communication présentée au XIXe Congrès International de Laiterie, NewDelhi 1974. 


\section{TABLEAU I}

Taux de pollution des laits de femme en France (novembre 71 à octobre 72) en $\mathrm{ppm}(\mathrm{mg} / \mathrm{kg})$ dans la matière grasse pure

\begin{tabular}{|c|c|c|c|}
\hline Pesticides & Moyenne & Minimum & Maximum \\
\hline $\mathrm{HCB}$ & 0,98 & $\begin{array}{c}0,50 \text { (Strasbourg } \\
\text { Bordeaux) }\end{array}$ & 3,50 (Lille) \\
\hline $\mathrm{HCH}$ total & 1,77 & 0,64 (Strasbourg) & 3,30 (St-Etienne) \\
\hline $\begin{array}{r}\mathrm{HCH} \alpha \\
\beta \\
\gamma\end{array}$ & $\begin{array}{l}0,040 \\
1,67 \\
0,06\end{array}$ & $\begin{array}{l}0,02 \\
0,84 \text { (Marmande) } \\
0,03 \text { (Bordeaux) }\end{array}$ & $\begin{array}{l}0,10 \text { (Lyon) } \\
3,70 \text { (Rouen) } \\
0,21 \text { (Paris) }\end{array}$ \\
\hline Heptachlore époxyde & 0,28 & 0,06 (Bordeaux) & 1,30 (Rouen) \\
\hline Dieldrine & 0,23 & 0,08 (St-Etienne) & 0,40 (Nantes) \\
\hline pp' DDE & 2,40 & 1,08 (Lille) & 6,10 (Bordeaux) \\
\hline pp' DDT & 0,84 & 0,34 (Paris) & 3,97 (Bordeaux) \\
\hline
\end{tabular}

\section{TABLEAU II}

Provenances des laits les plus pollués par catégorie d'insecticides (moyenne annuelle en $\mathrm{mg} / \mathrm{kg}$ )

\begin{tabular}{l|c|c|c|c}
\hline & HCH & $\begin{array}{c}\text { Heptachlore } \\
\text { époxyde }\end{array}$ & Dieldrine & DDT \\
\hline & & 0,53 & 0,24 & 3,40 \\
$\begin{array}{l}\text { Lille } \\
\text { Lyon }\end{array}$ & 2,13 & 0,43 & 0,24 & 3,58 \\
$\begin{array}{l}\text { Marmande } \\
\text { Nantes }\end{array}$ & 2,05 & 0,48 & 0,23 & \\
Paris & 2,38 & & 0,23 \\
Saint-Etienne & & & \\
Rouen & & &
\end{tabular}




\section{TABLEAU II (suite)}

Provenances des laits les moins pollués par catégorie d'insecticides (moyenne annuelle en $\mathrm{mg} / \mathrm{kg}$ )

\begin{tabular}{l|l|l|l|l}
\hline Bordeaux & 1,52 & & \\
Lyon & 1,46 & 0,22 & 0,22 & \\
Marmande & 1,24 & 0,13 & 0,18 & 2,16 \\
Saint-Etienne & & & \\
Strasbourg & & & & \\
\hline
\end{tabular}

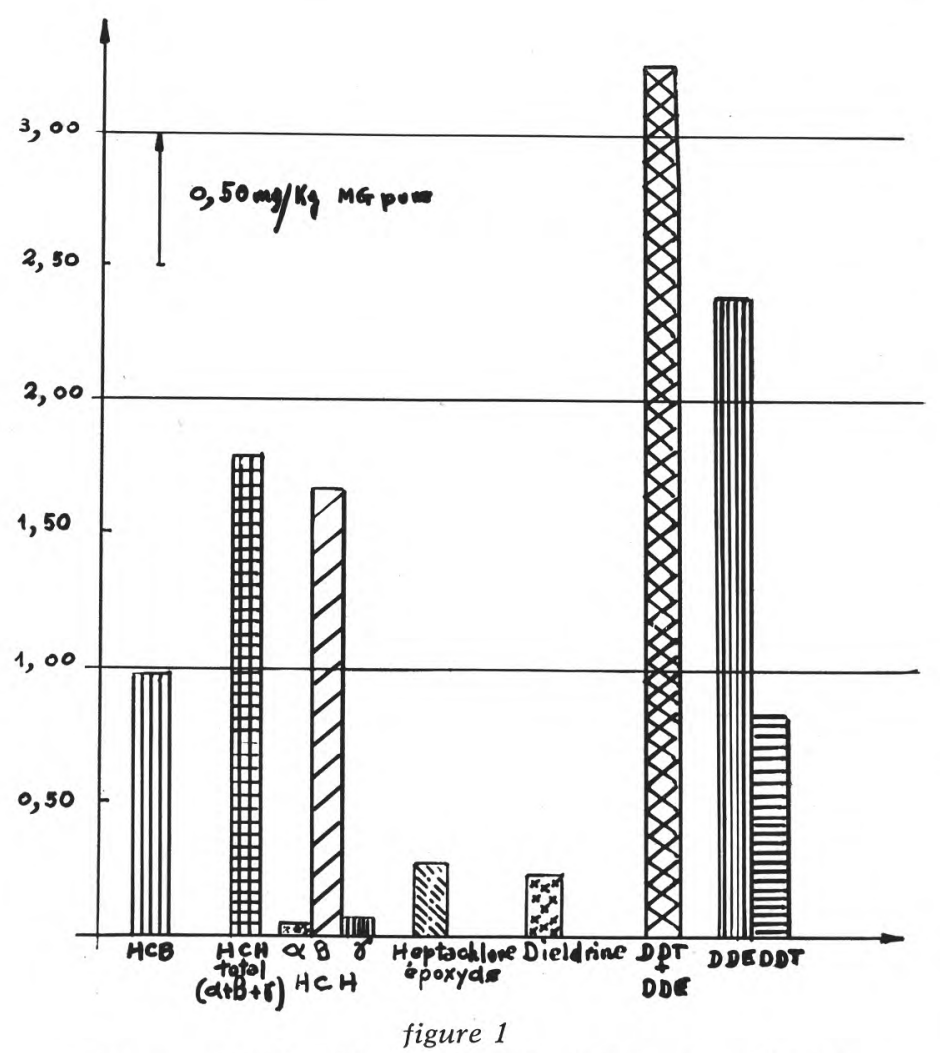

Taux moyen de la pollution des laits de femmes en France (novembre 1971 à octobre 1972) 
publiées) et des laits de femme montre que dans ces derniers les teneurs sont supérieures par rapport au lait de vache :

de 5 à 10 fois pour le $\mathrm{HCH}$

de 100 fois pour le DDT

de 4 à 5 fois pour l'Heptachlore époxyde

de 5 à 6 fois pour le Dieldrine

$4^{\circ}$ A l'inverse de ce qui se passe pour les laits d'origine animale, le taux de pollution ne présente pas de variations saisonnières.

$5^{\circ}$ Par contre, la pollution est variable qualitativement suivant la provenance géographique des échantillons (tableau II).

Ces différences tiennent peut-être au fait que le milieu social :t donc les habitudes alimentaires des nourrices approvisionnant les lactariums est variable : milieu urbain pour les lactariums de Bordeaux, Lyon et Paris.

$6^{\circ}$ Dans la région de Lille l'enquête a duré trois années. Les teneurs moyennes en $\mathrm{HCH}$ ont très sensiblement diminué ainsi que le montrent les chiffres ci-après : 2,65 en 1970 pour 1,85 en 1972.

\section{S u m m a r y}

Human milk in France is more polluted by pesticides residues than cow's milk. The main sources of pollution are DDT, its metabolites (pp' DDE and pp' DDT) and HCH.

No significant seasonal variations in the pollution have been found, but there are differences beetween samples coming from different regions of France.

\section{Bibliographie}

1. AcKer (L.), Schulte (E.) (1970). - Uber das Vorkommen von chlorierten Biphenylen und Hexachlorbenzol neben chlorierten Insektiziden in Humanmilch und menschlichem Fettgewebe. Naturwissenschaften. Dtsch., 57, 497.

2. ACKER (L.), SCHULte (E.) (1970). - Uber das Vorkommen chlorierter Kohlenwasserstoffe im menschlichen Fettgewebe und in Humanmilch. Deut. Lebensm. - Rundsch., 66, 385.

3. Curley (A.), Kimbrough (R.) (1969). - Chlorinated hydrocarbon insecticides in plasma and milk of pregnant and lactating women. Arch. environment. Health U.S.A., 18, 156.

4. Deichmann (W. B.), Mac Donald (W. E.) (1971). - Organochlorine pesticides and human health. Food Cosmet. Toxicol., G.B., 9, 91.

5. Fournier (E.) (1971). - Imprégnation par pesticides des êtres humains. Cah. Nut. Diét., 6, 31. 
6. Goursaud (J.), Luquet (F. M.), Casalis (J.) (1968). - Méthode rapide de contrôle de la teneur en résidus de quelques pesticides organochlorés dans les produits laitiers. Le Lait, 48, 645 .

7. Goursaud (J.), Luquet (F. M.), Casalis (J.) (1970). - Note sur le dosage des résidus de pesticides dans le lait et les produits laitiers. Rev. Lait. franç., $282,897$.

8. Goursaud (J.), Luquet (F. M.), Casalis (J.) (1972). - Dosage des résidus de pesticides dans les fromages : adaptation de la technique de la Food and Drug Administration. Rev. Lait. franç., 302, 779.

9. Gracheva (G. V.) (1970). - Excrétion de DDT dans le lait des mères allaitantes non exposées professionnellement au contact de cet insecticide. Vopr. Pitan., S.S.S.R., 6, 75.

10. HeyndRickx (A.), MAES (R.) (1969), - The excretion of chlorinated hydrocarbon insecticides in human mother milk. J. Pharm. Belg., 51, 459.

11. Kontek (M.), Kubacki (S.), Paradowski (S.), Wizrzchowieka (B.) (1971). Etude de la teneur du lait de femme en pesticides chloro-organiques. Pediatr. polska, Polska, 46, 183.

12. Luquet (F. M.), Goursaud (J.), Gaudier (B.) (1972). - Etude de la pollution des laits humains par les résidus de pesticides. Path. Biol., 20, 137.

13. Thakare (S. K.), Dewan (R. S.), Gulati (K. C.) (1969). - Build-up of DDT in human fat-oils and oilseeds as possible sources. Pesticides, India, 3, 13.

14. Truhaut (R.) (1966). - Problèmes toxicologiques posés par l'emploi des pesticides en agriculture. Bull. I.N.S.E.R.M., 21, 1063.

15. Tuinstra (L. G. M. T.) (1971). - Organochlorine insecticide residues in human milk in the Leiden région. Netherl. Milk Dairy J., 25, 24.

16. Unterman (W. H.), Sirghie (E.) (1969). - Impregnarea organismului uman cu organoclorate. Cercetarea continutului de DDT si DDE în laptele matern. Igiena, Român., 18, 221. 